\title{
Intelligent Community Control Network Based on Wireless IP Service
}

\author{
Zhenjia Chen, Yonghui Zhang, Xia Guo
}

\author{
College of Information Science \& Technology, Hainan University, Haikou 570228, China \\ E-mail: zhyhemail@163.com
}

\begin{abstract}
Keywords: Control Network, Control Message, IP Service and Time Delay
Abstract. Control Network (CN) has been developed in many field. The application of intelligent community has no far from our life. In this paper, we propose the establishment of $\mathrm{CN}$ based on wireless IP service. Common device can access network rapidly through wifi or Ethernet. The development of application can be released quickly. 5.8G technology is as the core network of CN to realize high data throughout. The node of network encapsulates heterogeneous interface to realize data transmission. The control terminal can be merged into $\mathrm{CN}$ with minimum modify. The node in $\mathrm{CN}$ is as the router to transfer data packet, and is as the processing unit to monitor the quality of network. The network switches the strategy to adapt current circumstance based on the variation of time delay and the priority of control message. In this article, zigbee IP is suggested to be the RF module of control terminal. Compared with wired measurement and control system of the traditional control network, this system can be networking flexibly. It reduces the cost of the control network. It is an effective and intelligent wireless network solutions.
\end{abstract}

\section{Introduction}

Nowadays, consumer electronics have be seen everywhere in human`s life. Every sensor network and intelligent equipment let our life become more convenient. In the global, cable is replaced by wireless that has become an irreversible trend,it is more obvious in the field of Internet of Things (IoT). wifi and zigbee technology have been very popular in control and management of smart city. wifi technology has the advantage of easy to develop, the product cost is low. Many start-ups develop intelligent home products based on wifi technology, but its shortcoming is also obvious. First of all, the biggest problem of wifi is that the security is very low, the stability of wireless product is poor. Foreign media had reported the intelligent household products was easily breached by hacker. And then American hacker showed how to get all information by unmanned drone from mobile phone by wifi. Secondly, the high power consumption is also a great weakness toward wifi, leading to that its application has been limited in the field of intelligent household. Due to its high power consumption, wifi will not be used in areas such as intelligent door lock, infrared forward control unit, various sensors and other products. In addition, the wifi network capacity is low and it is restricted to expand. At present, the size of the wifi network is generally not more than 16 equipment, and the number of home appliance in the family, such as the ordinary switch, electric light, has been far more than 16. Apparently intelligent household based on wifi technology is limited in the future.

Relative to the wifi technology, the advantage of zigbee is obvious. zigbee technology has high security in short range wireless communication. zigbee technology adopted extremely low power design theory. The actual application of a battery can use 2 years or so. This low-power technology has a wide range of applications in the field of intelligent household. [1] proposed the zigbee network applications achieving energy saving in the heating system. [2] describes that the zigbee network performance is superior. In theory, a zigbee gateway can connect more than 65000 equipment. At present, the stable network is composed of more than 100 kinds of equipment. The network scale is far than wifi, Z-Wave, Bluetooth technology and so on. zigbee alliance announced that the third set of specifications zigbee IP has been developed and introduced to the public. zigbee IP is the first open standard for an IPv6-based full wireless mesh networking solution and provides seamless Internet connections to control low-power, low-cost devices. zigbee IP specifications joined the network and security layer and an application framework. The IEEE802.15.4 standard will be improved. zigbee IP provides an extensible structure with end-to-end IPv6 networking ability without gateway. Thus, it 
laid a foundation for the development of the IoT. It means that we can realize large-scale CN based on IP layer. [3] combined 802.15.4 with zigbee to implement a smart home energy management system. [4] proposed wireless sensor network, collecting the temperature and humidity data in the greenhouse and sending to the coordinator in a wireless manner. [5] proposed to combine the zigbee network with the IPv4 / IPv6 network to realize the remote monitoring network

However, the communication distance of zigbee is short, penetrability is weak. The thickness of the barrier seriously affect the communication distance. It is suitable for small range of large-scale wireless CN. zigbee and wifi also work in ISM $2.4 \mathrm{GHz}$. The band has been very crowded and spectrum interference is very serious. We can use the heterogeneous network architecture to combine the advantages of each network and apply to the intelligent community. Consider [6] and [7], we use wireless mesh network (WMN) to set up distributed control node.

In this paper, we propose zigbee network as a sensor network and wireless wifi network as the monitoring or user access, $5.8 \mathrm{G}$ technology as the core network for data aggregation. $5.8 \mathrm{GHz}$ has the advantage of high transmission rate and broadband communication. It can satisfy the requirement of the vast amount of intelligent data. The network establishes wireless mesh heterogeneous communication. It realizes wireless intelligent monitoring and control based on IP services.

\section{Network Structure}

The intelligent community is compose of many sub-module. Each sub-module implements a specific control function and acts as a node that transmits packets to the core network. Sub-module acts as the gateway of heterogeneous networks. During zigbee IP specifications joining the network layer, the fusion of IPv6 and zigbee has been applied. Control Terminal (CT) shares the network resources. They organize the intelligent community control data and status information. The message is passed between the wireless network and gateway through short-range wireless communication.

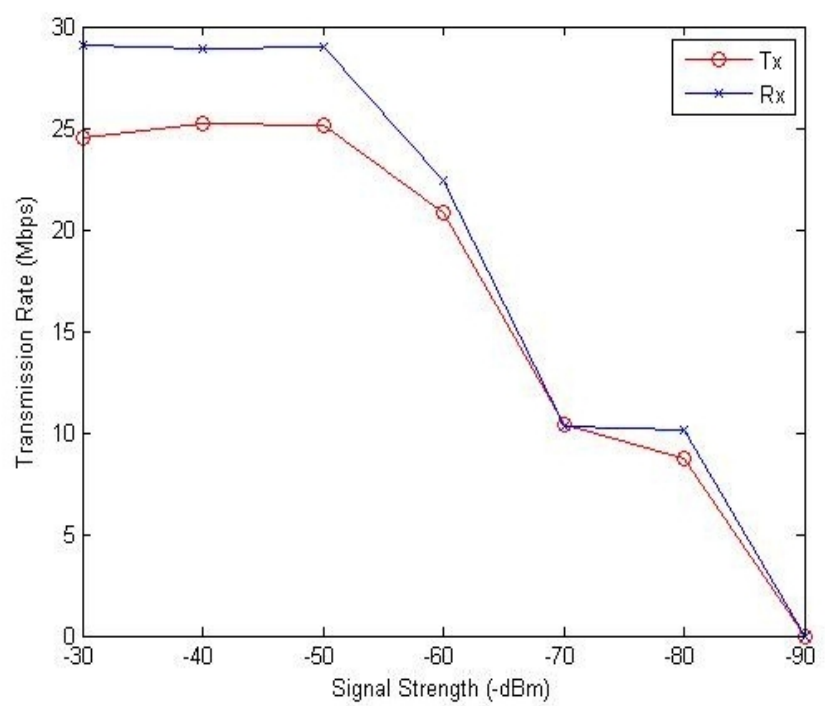

(a) One-way transmission

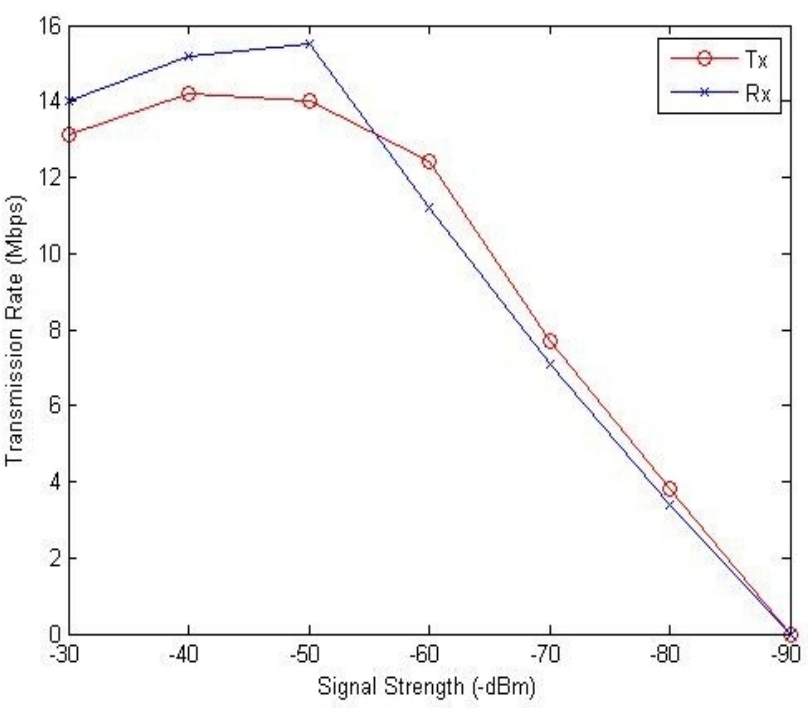

(b) Two-way transmission

Fig 1. The throughput data of core network in one-way transmission. The transmission rate decreases as the weakening of the signal. The transmission rate of Rx is few higher than Tx. In two-way transmission. Since $-60 \mathrm{dBm}$, the transmission rate declines linearly.

Terminal control node based on IP service would be proposed in the system. Large-scale terminal could create huge throughput. So we choose $5.8 \mathrm{GHz}$ technology as the core network. Using the Router OS of $5.8 \mathrm{GHz}$ devices to build WMN, it realizes wireless large capacity up-link and down-link. The throughput data of core network is shown in Figure 1.

The data of CN contain multimedia and plentiful command. So this system must satisfy broadband service.According to the definition of International Mobile Telecommunications Advanced (IMT-Advanced), services are divided into five types of services. As shown in Table 1. 
Table1. Service Types

\begin{tabular}{c|c}
\hline \hline Type & Peak Bit Rate \\
\hline Very low speed data & $<16 \mathrm{~kb} / \mathrm{s}$ \\
\hline Low speed data & $<144 \mathrm{~kb} / \mathrm{s}$ \\
Low speed multimedia & $<2 \mathrm{Mb} / \mathrm{s}$ \\
\hline Medium speed multimedia & $<30 \mathrm{Mb} / \mathrm{s}$ \\
\hline High speed multimedia & $30 \mathrm{Mb} / \mathrm{s}-100 \mathrm{Mb} / 1$ \\
\hline Ultra high speed & $\mathrm{Gb} / \mathrm{s}$ \\
multimedia & $25 \mathrm{Mb}$ \\
\hline \hline
\end{tabular}

Compared with Figure 1, one-way throughput can reach 25Mbps when the strength of signal is strong $(>-50 \mathrm{dBm})$. In two-way transmission, the rate would be half of one-way transmission. When the strength of signal is $-80 \mathrm{dBm}$, the one-way transmission rate can reach $10 \mathrm{Mbps}$, the two-way rate can reach $4 \mathrm{Mbps}$. When the strength of signal is above $-90 \mathrm{dBm}$, the system cannot maintain data link and lose all packet.According to the data, we can discover the core network can meet medium speed multimedia service. Compared with the definition of IMT-Advanced, the core network satisfies high speed multimedia service. It can meet large-scale terminal access. In order to satisfy user`s devices access, the network merge wifi and Ethernet interface together. In some public places, image catching is required. The network can equips IP Camera to realize monitoring. Some processes should be implemented at local communication node in order to achieve real-time operation. So the local node not only acts as the router, also as the processor for control mission. The structure diagram of $\mathrm{CN}$ is shown in Figure 2.

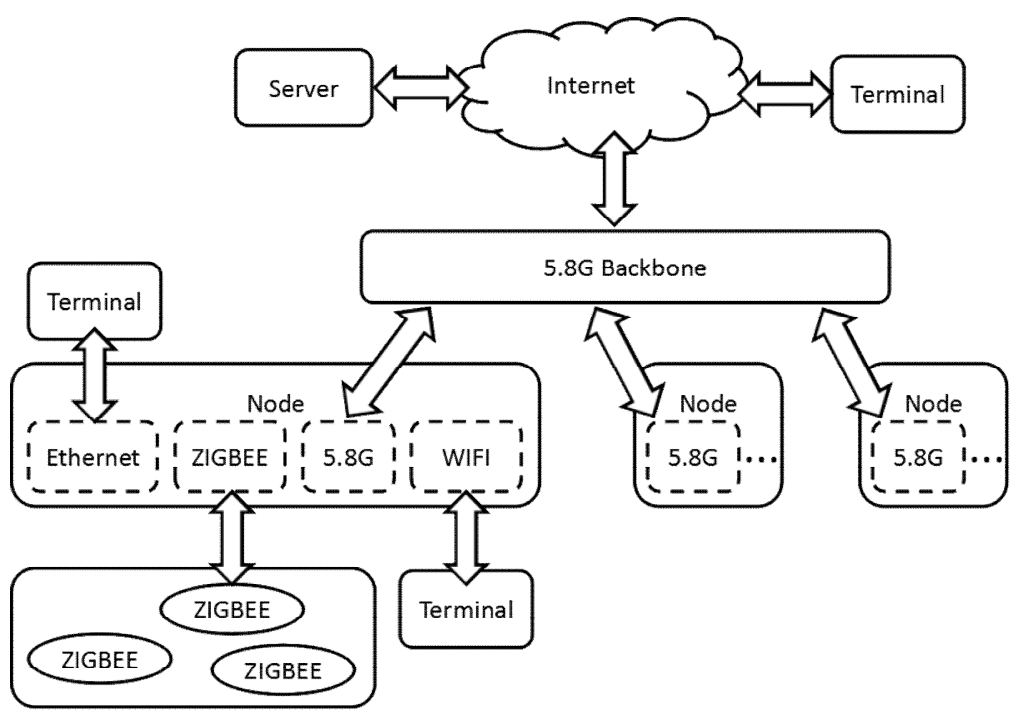

Fig 2. The structure diagram of $\mathrm{CN}$. The core network satisfy huge throughout. The local communication node implements heterogeneous network communication as a router.

The node transmits data packet as a router through multiple heterogeneous interface. By the way, node can also process local data automatically and work as the distributed equipment. Every node in wireless mesh network divert calculation pressure away from server.The server acts as the process center of big data and provides information service for terminals. The terminal that accesses network through Internet can control every point remotely. Nodes collect control command or basis data through the Internet. Local node can provides general interface for common mobile devices access.

\section{The Structure of Node}

In Figure 2, zigbee interface is as the gateway of $\mathrm{CN}$. There are many other standard buses can access CN, such as Universal Serial Bus (USB), Controller Area Network (CAN), RS-485. Every bus acts as the interface of node. The node realizes open system interconnection reference model and transfers 
data packet to IP layer. It is convenient to develop application in user layer. The structure of node is shown in Figure 3.

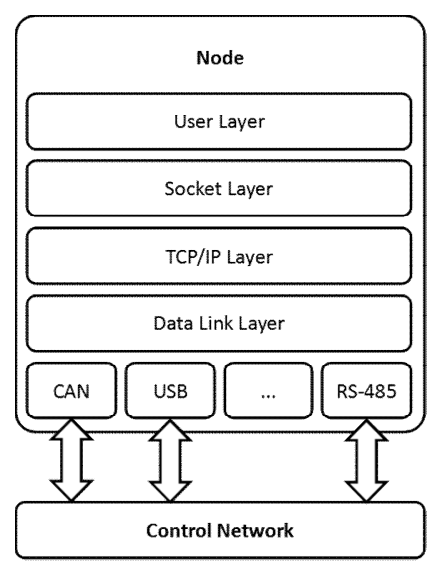

Fig 3. The structure diagram of node. The node transfers data packet through multiple heterogeneous interface as a router, and integrates data with different format.

Local node has routing list for local wireless network. Different bus is encapsulated to unified standards. It is not necessary for node or terminal to understand the principle of bus and the hardware. It is the work of abstraction layer between data link layer and bus. Basic information for every CT is composed of IP address and control commands. We call those data packet in CN as Control Message (CM). There are two kinds of message for terminals, one is receiving CM, other one is sending CM. Each message contain two frames. The structure of receiving CM is shown in Figure 5. Receiving CM contain current status information from source CT. The source IP marks unique one CT in the network. The device description is as one parameter in second frame. Receiving CM from CT distributes status information through multicast. Terminal can get the information of CT from receiving $\mathrm{CM}$ and decodes the packet.

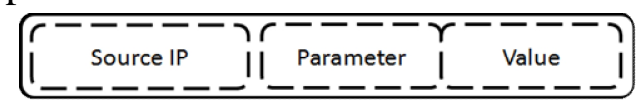

Fig 4. The structure diagram of receiving CM. The first frame of CM is source IP. The second frame is the status information data of CT.

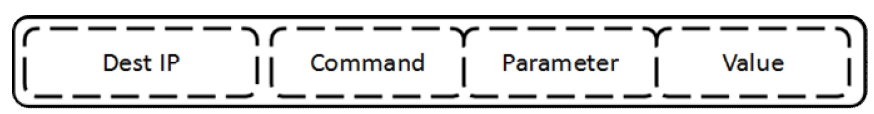

Fig 5. The structure diagram of sending CM. The first frame of CM is destination IP. The second frame is the command content for target CT.

Base on the information of CT, terminal can operates the CT through sending CM. The structure diagram of sending CM is shown in Figure 5. Through destination IP, terminal establishes connection by socket and realizes end-to-end control. The process of end-to-end control is shown in Figure 6. Nodes compose the core network of CN. They transmit CM between CT and terminal. First, the node receives multicast $\mathrm{CM}$ from $\mathrm{CT}$, and transmits it to terminal. Terminal decodes the packet and establishes connection with the node. After verifying the signal of access success, terminal starts to send command and receives status data from CT. 


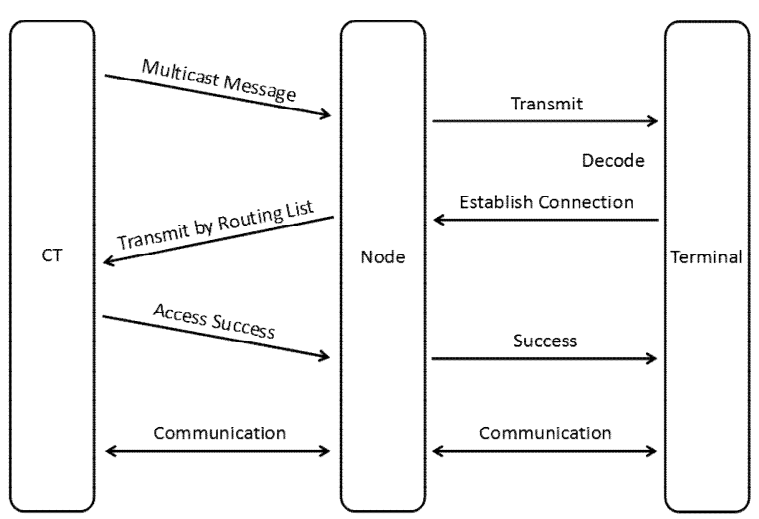

Fig 6. The process of end-to-end control. First, CT broadcast the CM. The CM contain the information of CT.The node transmit data packet as the router or process the data locally.

The length of IPv6 is 128bit. The length of CM here is determined by the quantity of command. We use group average length 128Byte as the length of second frame. The specification of CM is shown in Figure 7.

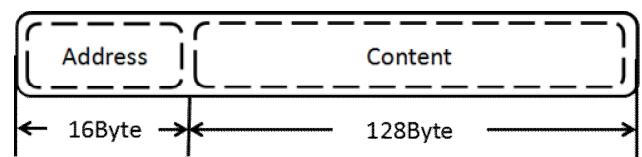

Fig 7. The specification of CM. The length of the second frame refers to group average length in TCP/IP. The total length is 144Byte.

In $\mathrm{CN}$, there are vast $\mathrm{CMs}$ at the same time. [8] focused on the influence of packet loss on the control system. Based on the switching system, they carried out the controller design. But the packet loss happen in unstable network circumstance. We can enhance signal strength or optimize network topological structure to resolve this problem. [9] proposed TCP and UDP time delay model of Internet network control system based on data stream. They referred to Transit-Stub structure and put forward the time delay measure model. CM has the advantage of high timeliness, small data packet. In general, this article suggests the transmission of CM through TCP. TCP is the reliable communication protocol and is very suitable for $\mathrm{CN}$. When network happen blocking and the tine delay increase, the node would switch UDP to transmit CM. Outdated CM would be abandoned in the network. The network allow higher priority packet to be transmitted first.

$\mathrm{CN}$ need short delay and fast reaction. Based on the variation of RF module signal in core network, we test time delay and packet loss probability in huge-capacity (65500Byte). When the signal below $-90 \mathrm{dBm}$, the packet loss probability reach $100 \%$. At this time, the network cannot transmit CM normally. The time delay increases when signal recede. The test result is shown in Figure 8.

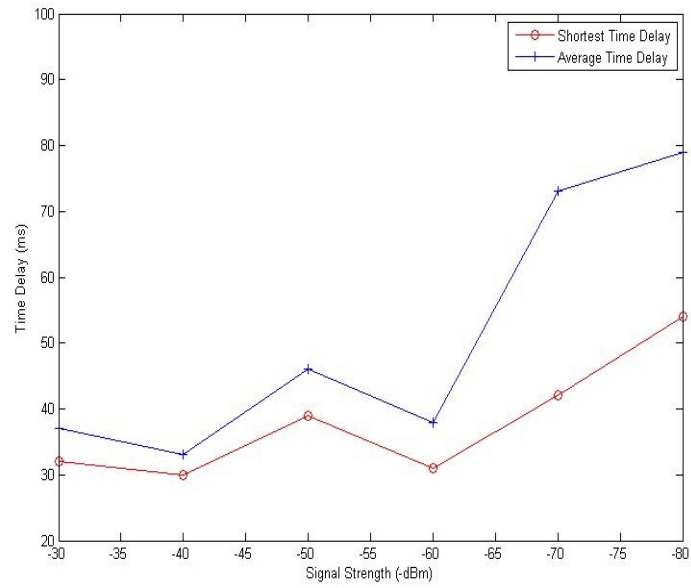

Fig 8. The time delay of core network in huge-capacity. Test the concurrent throughput of CN through TCP at the same time. When the signal is good (Above $-60 \mathrm{dBm})$, the network keep the time delay in $30 \mathrm{~ms}$ to $40 \mathrm{~ms}$. 
The node maximizes reducing time delay through switch transmission protocol. It refers to the variation of time delay $T_{d}$ and packet loss probability $P_{l}$. In $\mathrm{CN}$, other import parameter is prior to CM. First in this article, the network set a threshold for time delay $V_{T}$ (Refer to bad signal point as -60dBm) and for packet loss probability $V_{P}$ (Fail to maintain physical link as $-90 \mathrm{dBm}$ ) based on the quality of network.

$$
\mathrm{T}_{d}<V_{T} .
$$

When the physical link can guarantee stable connection in good signal as formula (1), the network uses TCP to maintain every CM to arrive target terminal. Especially, some monitor application, such as security and protection, need multimedia communication. That would occupy huge capacity.

$$
\left\{\begin{array}{l}
\mathrm{T}_{d}>V_{T} \\
P_{l}<V_{P}
\end{array} .\right.
$$

During increasing number of CT, signal interference between wireless devices would influence the quality of signal. In the above test data, it is the situation of bad signal and $P_{l}$ can meet the demand of network. As formula (2). With the time delay increasing, the number of CM would decrease. We propose refer to the priority of $\mathrm{CM}$ to switch transmission protocol. We propose that the transport protocol should be switched with reference to the priority of the CM. The CM with high priority such as warning message should transfer through TCP. The CM with low priority such general status data should transfer through UDP. The outdated CM would be discarded after be detected.

$$
\mathrm{P}_{l}>V_{P} \text {. }
$$

When the network is in very unstable status, the increasing of $P_{l}$ would lead to CM loss. As formula (3). The network should use UDP to maintain bottommost connection. In addition, the node shuts down the multimedia source such as IP Camera through IP control model so that reduces the pressure of network. The structure of IP control model is shown in Figure 9.

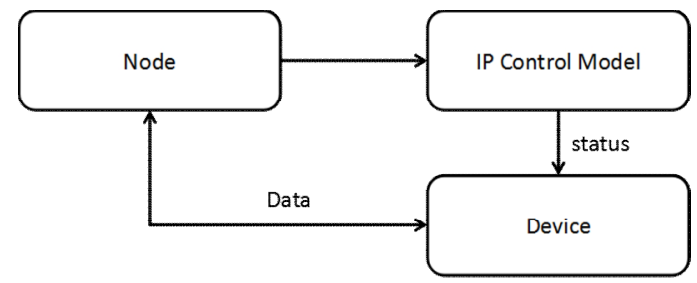

Fig 9. IP control model. After detecting the quality of network, node decides whether turn off the device through IP control model. The network control device by the energy saving policy in particular.

\section{Control Network}

In this article, we design the control network based on zigbee wireless network. The zigbee network adopts the star topology. The network is composed of coordinator and sensor nodes. Device set parameter and transfer data with AT instruction set through serial port. AT in here is encapsulated to CM. The node of core network transforms the AT between sensor nodes and user terminal. The structure of coordinator and sensor nodes is shown in Figure 10.

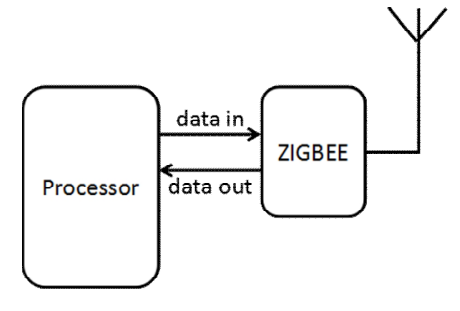

Coordinator

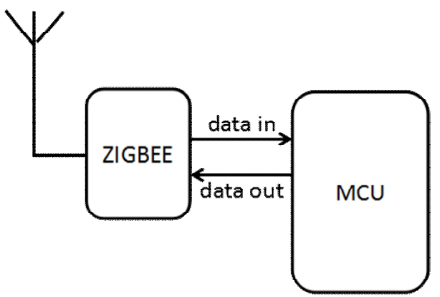

Sensor Node

Fig 10. Coordinator and sensor nodes. The processor is as equal to the node in CN. The MCU of sensor node realizes different function. 
MCU contain some special senor, such as humidity sensor, temperature sensor, ozone sensor, lumen sensor, etc. Those sensors get the status information of environment. The terminal can maximize restoring the scene according to all kind of parameters. Based on IPv6, zigbee IP is the wireless mesh network solution of open standards. The node accesses zigbee model by IP. For whole wireless network, the system uses zigbee model to decrease power consumption and provides seamless Internet connection.

\section{Conclusions}

Intelligent community control network based on wireless IP service realizes standard OSI model. User Terminals send and receive CM through TCP/IP. On the basic of IP layer, the application development is very convenient. The node encapsulates heterogeneous interface to realize data transmission. It is as the router, or is as the processing unit to monitor network and realize local calculation. In this article, we propose that using $5.8 \mathrm{G}$ interface as the core network realizes high data throughout. It can satisfy high speed multimedia service. Especially in the intelligent community control network, there is a mount of CTs in network. By the way, this network model can be also used in industrial control with multiple terminal. The general standard make every common devices access network rapidly with less modification.

In future, we would consider to use MIMO to increase the throughout of core network, optimize the property of node to reduce the time delay of data packet transmission. Through researching wireless networking protocol, the quality of the communication network would be improved. Through the quality and the parameter of network, we would modify the strategy to avoid network congestion, improve the immediacy of interaction. Compared with wired measurement and control system of the traditional control network, this structure reduces the cost of the network and provides an effective intelligent wireless network solutions.

\section{Acknowledgements}

In this paper, the research was sponsored by the National Natural Science Foundation of China (Grant No. 61561018), the Application Technology Research and Development and Demonstration of Hainan Province (Grant No. ZDXM2015104), the CERNET Innovation Project (Grant No. NGII20160319) and the Scientific Research Project of Higher Education in Hainan Province (Grant No. Hnky2015ZD-6).

The corresponding author is Yonghui Zhang.

\section{References}

[1] M. Cagnetti, F. Leccese, A. Proietti, Energy Saving Project for Heating System with zigbee wireless control network[C], 2012 11th International Conference on Environment and Electrical Engineering (EEEIC), 2012.3.

[2] zigbee Alliance, zigbee Specification.version r17, 2008.1.

[3] Dae-Man Han, Jae-Hyun Lim, Smart Home Energy Management System using IEEE 802.15.4 and zigbee[J], IEEE Transactions on Consumer Electronics, Vol.56, No.3, 2010.8.

[4] LUO Quan, QIN Linlin, LI Xiaofeng, WU Gang, The implementation of wireless sensor and control system in greenhouse based on zigbee[C], Proceedings of the 35th Chinese Control Conference, 2016.7.

[5] Fang Tao, the remote monitoring network communication based on zigbee and IPv6[D], Beijin University of Technology, 2013.6. 
[6] Fang, The Wireless Control Network: A New Approach for Control Over Networks[J], IEEE TRANSACTIONS ON AUTOMATIC CONTROL, Vol.56, No.10, 2011.10.

[7] Kaushik Nanda, Kiran Nayak, Shrikrishna Chippalkatti, Ramana Rao, David Selvakumar, Haribabu Pasupuleti, Web based Monitoring and Control of WSN using WINGZ (Wireless IP Network Gateway for zigbee)[C], 2012 Sixth International Conference on Sensing Technology (ICST), 2012.

[8] CHEN Qing, WU Min, Design of networked controller for systems with packet dropout and networked-induced delays, Control and Decision, Vol.26, No.2, 2011.2.

[9] CHENG Lun, WANG Zhong-jie, Research on time-delay model for NCS based on data streams, Control and Decision, Vol.26, No.4, 2011.4. 